East Asian Mathematical Journal

\title{
REMARK ON THE CONTROLLABILITY FOR SEMILINEAR EVOLUTION EQUATIONS
}

\author{
Jin-Mun JEONG
}

\begin{abstract}
In this paper we deal with approximate controllability for semilinear system in a Hilbert space. In order to obtain the controllability, we assume that the system of the generalized eigenspaces of the principal operator is complete in the state space, which has a simple form and can be applied to many examples. Because of its simple form, some examples of controllability of the systems governed by the semilinear equations will be given.
\end{abstract}

\section{Introduction}

Let $H$ and $V$ be complex Hilbert spaces such that the imbedding $V \subset H$ is compact. The inner product and norm in $H$ are denoted by $(\cdot, \cdot)$ and $|\cdot|$, and those in $V$ are by $((\cdot, \cdot))$ and $\|\cdot\|$. Let $-A$ be the operator associated with a bounded sesquilinear form $a(u, v)$ defined in $V \times V$ and satisfying Gårding inequality

$$
\operatorname{Re} a(u, v) \geq c_{0}\|u\|^{2}-c_{1}|u|^{2}, \quad c_{0}>0, \quad c_{1} \geq 0
$$

for any $u \in V$. That is, $A$ is the self adjoint operator defined by

$$
(A u, v)=-a(u, v), \quad u, v \in V .
$$

Then we know that $A$ generates an analytic semigroup in both of $H$ and $V^{*}$ where $V^{*}$ stands for the dual space of $V$.

The object of this paper is to investigate the quality of reachable set of the following semilinear parabolic type equation

$$
\left\{\begin{array}{l}
\frac{d}{d t} x(t)=A x(t)+f(t, x)+B u(t), \quad t \in(0, T], \\
x(0)=x_{0} .
\end{array}\right.
$$

The existence and uniqueness of solution of the above system are proved in $[1$, $2,5]$. The condition for equivalence between the reachable set of the semilinear system and that of its corresponding linear system(the case where $f(\cdot, \cdot)=0$

Received May 8, 2013; Accepted October 25, 2013.

2000 Mathematics Subject Classification. 93C23,34K40.

Key words and phrases. controllability, semilinear evolution equation, generalized eigenspace, complete, controller operator.

This work was supported by a Research Grant of Pukyong National University(2013). 
in (1.1)) was established in $[5,9]$. This paper is dealt with another applicable condition for controller of approximate control problem.

The main result in this paper will show a sufficient condition for approximate controllability obtained in [5] for the system(1.1) with some conditions for the operator $A$. In order to obtain the controllability, we need some conditions of a range condition of the control action operator and the completeness of the generalized eigenspaces of the principal operator in the state space, which has a simple form and can be applied to many examples. Moreover, we introduce the solution semigroup and provide the representations of spectral projections by using the spectral properties of the operator $A$. Because of its simple form, some examples of controllability of the systems governed by the semilinear equations will be given.

\section{Main results}

Let $\mathrm{U}$ be a Banach space of control variables and the controller $B$ be a bounded linear operator from $U \subset H$ to $H$. Let $f$ be a nonlinear mapping $\mathbb{R} \times V$ into $H$. Hence, we assume more general Lipschitz condition: for any $x_{1}, x_{2} \in V$ there exists a constant $L>0$ such that

$$
\left\{\begin{array}{l}
\left|f\left(t, x_{1}\right)-f\left(t, x_{2}\right)\right| \leq L|| x_{1}-x_{2}||, \\
f(t, 0)=0
\end{array}\right.
$$

Then we first introduce the regularity of solutions of the semilinear equation (1.1).

Proposition 2.1. Under the assumptions (2.1), there exists a unique solution of (1.1) such that

$$
x \in L^{2}(0, T ; V) \cap W^{1,2}\left(0, T ; V^{*}\right) \subset C([0, T] ; H)
$$

for any $x_{0} \in H$. Moreover, there exists a constant $C$ such that

$$
\|x\|_{L^{2}(0, T ; V) \cap W^{1,2}\left(0, T ; V^{*}\right)} \leq C\left(\left|x_{0}\right|+\|u\|_{L^{2}(0, T ; U)}\right),
$$

where

$$
\|\cdot\|_{L^{2}(0, T, V) \cap W^{1,2}\left(0, T ; V^{*}\right)}=\max \left\{\|\cdot\|_{L^{2}(0, T ; V)},\|\cdot\|_{W^{1,2}\left(0, T ; V^{*}\right)}\right\} .
$$

Let $x(T ; f, u)$ be a solution of the system (1.1) associated with nonlinear term $f$ and control $u$ at time $T$. We define reachable sets for the system (1.1) as follows:

$$
\begin{aligned}
& L_{T}=\left\{x(T ; 0, u): u \in L^{2}(0, T ; U)\right\}, \\
& R_{T}=\left\{x(T ; f, u): u \in L^{2}(0, T ; U)\right\} .
\end{aligned}
$$

It is known that $L_{T}$ is independent of $T$ (see [7, Lemma 7.4.1]). 
In virtue of the Riesz-Schauder theorem, if the imbedding $V \subset H$ is compact then the operator $A$ has discrete spectrum

$$
\sigma(A)=\left\{\mu_{n}: n=1,2, \ldots\right\}
$$

which has no point of accumulation except possibly $\mu=\infty$ (see $[6,8]$ ). Let $\mu_{n}$ be a pole of the resolvent of $A$ of order $k_{n}$ and $P_{n}$ the spectral projection associated with $\mu_{n}$

$$
P_{n}=\frac{1}{2 \pi i} \int_{\Gamma_{n}}(\mu-A)^{-1} d \mu,
$$

where $\Gamma_{n}$ is a small circle centered at $\mu_{n}$ such that it surrounds no point of $\sigma(A)$ except $\mu_{n}$ (cf. Nakagiri [4]). Then the generalized eigenspace corresponding to $\mu_{n}$ is given by

$$
H_{n}=P_{n} H=\left\{P_{n} u: u \in H\right\},
$$

and we have that from $P_{n}^{2}=P_{n}$ and $H_{n} \subset V$ it follows that

$$
P_{n} V=\left\{P_{n} u: u \in V\right\}=H_{n} .
$$

Let us set

$$
Q_{n}=\frac{1}{2 \pi i} \int_{\Gamma_{n}}\left(\mu-\mu_{n}\right)(\mu-A)^{-1} d \mu .
$$

Then we remark that $\operatorname{dim} H_{n}<\infty$ and

$$
Q_{n}^{i}=\frac{1}{2 \pi i} \int_{\Gamma_{n}}\left(\mu-\mu_{n}\right)^{i}(\mu-A)^{-1} d \mu .
$$

It is also well known that $Q_{n}^{k_{n}}=0$ ( nilpotent) and $\left(A-\mu_{n}\right) P_{n}=Q_{n}$.

Definition 1. The system of the generalized eigenspaces of $A$ is complete in $H$ if $\mathrm{Cl}\left\{\operatorname{span}\left\{H_{n}: n=1,2, \ldots\right\}\right\}=H$ where $\mathrm{Cl}$ denotes the closure in $H$.

Let $S(t)$ be an analytic semigroup generated by $A$. Then the mild solution of (1.1) is represented by

$$
x(t ; f, u)=S(t) x_{0}+\int_{0}^{t} S(t-s)\{f(s, x(s)+B u(s)\} d s .
$$

We denote the bounded linear operator $\hat{S}$ from $L^{2}(0, T ; H)$ to $H$ by

$$
\hat{S} p=\int_{0}^{T} S(T-s) p(s) d s
$$

for $p \in L^{2}(0, T ; H)$.

Definition 2. The system (1.1) is approximately controllable on $[0, T]$ if $\overline{R_{T}}=$ $H$, that is, for any $\epsilon>0$ and $z \in H$ there exists a control $u \in L^{2}(0, T ; U)$ such that

$$
\left|z-S(T) x_{0}-\hat{S}\left\{f\left(\cdot, x_{u}(\cdot)\right)+B u\right\}\right|<\epsilon .
$$


We need the following hypotheses:

(A) The system of the generalized eigenspaces of $A$ is complete.

(B) For any $\epsilon>0$ and $p \in L^{2}(0, T ; H)$ there exists a $u \in L^{2}(0, T$; U) such that

$$
\|S(t-\cdot)(p(\cdot)-B u(\cdot))\|_{L^{2}(0, t ; H)} \leq \epsilon, \quad 0 \leq t \leq T .
$$

Proposition 2.2. Under the assumption (B), we have $\overline{L_{T}}=H$ (cf. [3]).

Theorem 2.3. Let us assume the hypotheses (A) and (B). Then we have $\overline{R_{T}}=\overline{L_{T}}$ for any $T>0$.

In virtue of Proposition 2.2 and Theorem 2.1 we have known that the system (1.1) is approximately controllable in conclusion.

Remark 1. If the semigroup $S(t)$ generated by $A$ is compact, we may assume the condition (B) at only time $T$, that is, we can rewrite the condition (B) as follows.

For any $\epsilon>0$ and $p \in L^{2}(0, T ; H)$ there exists a $u \in L^{2}(0, T ; U)$ such that

$$
|\hat{S} p-\hat{S} B u|<\epsilon \text {. }
$$

Remark 2. In Naito [3] he proved Theorem 2.2 under assumptions (B) and compact operator $S(t)$ and also Zhou in [10] showed it under assumption (B) and another condition of range of controller.

\subsection{Proof of main results}

First of all, for the meaning of assumption (B) we need to show the existence of controller satisfying $\mathrm{Cl}\left\{B u: u \in L^{2}(0, T ; U)\right\} \neq L^{2}(0, T ; H)$. In fact, Consider about the controller $B$ defined by

$$
B u(t)=\sum_{n=1}^{\infty} u_{n}(t),
$$

where

$$
u_{n}= \begin{cases}0, & 0 \leq t \leq \frac{T}{n} \\ P_{n} u(t), & \frac{T}{n}<t \leq T .\end{cases}
$$

Hence we see that $u_{1}(t) \equiv 0$ and $u_{n}(t) \in \operatorname{Im} P_{n}$. By completion of generalized eigenspaces of $A$ we may write that $g(t)=\sum_{n=1}^{\infty} P_{n} g(t)$ for $g \in L^{2}(0, T ; H)$. Let us choose $g \in L^{2}(0, T ; H)$ satisfying

$$
\int_{0}^{T}\left\|P_{1} g(t)\right\|^{2} d t>0 .
$$

Then since

$$
\begin{aligned}
& \int_{0}^{T}\|g(t)-B u(t)\|^{2} d t=\int_{0}^{T} \sum_{n=1}^{\infty}\left\|P_{n}(g(t)-B u(t))\right\|^{2} d t \\
& \geq \int_{0}^{T}\left\|P_{1}(g(t)-B u(t))\right\|^{2} d t=\int_{0}^{T}\left\|P_{1} g(t)\right\|^{2} d t>0,
\end{aligned}
$$


the statement mentioned above is reasonable.

The proof of Proposition 2.1 is from Theorem 3.1 in [5].

Proof of Proposition 2.2. Let $x_{0} \in D(A)$, Then putting $h(s)=\left(x_{0}+\right.$ $\left.s A x_{0}\right) / t$ it follows that

$$
x_{0}=\int_{0}^{t} S(t-s) h(s) d s .
$$

Thus by the condition (B) there exists $u \in L^{2}(0, T ; U)$ such that

$$
\left\|x_{0}-\int_{0}^{t} S(t-s) B u(s) d s\right\|<\epsilon .
$$

Therefore, the density of the domain $D(A)$ in $H$ implies approximate controllability of (1.1) and (1.3), the proof of Proposition 2.2 is complete.

Proof of Theorem 2.3. Through this section, we assume that the system of the generalized eigenspaces of $A$ is complete. Then we will prove that the assumptions (A) and (B) are a sufficient condition for the following statement $(\mathrm{H})$ in Theorem 4.1 as in [11]:

(H) For any $\epsilon>0$ and $p \in L^{2}(0, T ; H)$ there exists a $u \in L^{2}(0, T ; U)$ such that

$$
\left\{\begin{array}{l}
\left.\int_{0}^{t} S \mid t-s\right) p(s) d s-\int_{0}^{t} S(t-s) B u(s) d s \mid<\epsilon, \quad 0 \leq t \leq T, \\
\|B u\|_{L^{2}(0, T ; H)} \leq q\|p\|_{L^{2}(0, T ; H)} .
\end{array}\right.
$$

where $q$ is a constant independent of $p$.

If $\mu_{n} \in \sigma(A)$ then we have the Laurent expansion for $R(\mu-A) \equiv(\mu-A)^{-1}$ at $\mu=\mu_{n}$ whose principal part ( the part consisting of all the negative power of $\left.\left(\mu-\mu_{n}\right)\right)$ is a finite series:

$$
R(\mu-A)=\frac{P_{n}}{\mu-\mu_{n}}+\sum_{i=1}^{k_{n}-1} \frac{Q_{n}^{i}}{\left(\mu-\mu_{n}\right)^{i+1}}+R_{0}(\mu),
$$

where $R_{0}(\mu)$ is a holomorphic part of $R(\mu-A)$ at $\mu=\mu_{n}$.

Since the system of generalized eigenspaces of $A$ is complete, it holds that for any $\epsilon>0$

$$
\left|g(s)-\sum_{n=1}^{\infty} P_{n} g(s)\right| \leq \epsilon
$$

for $g \in L^{2}(0, T ; H)$. For the sake of simplicity, we assume that $S(t)$ is uniformly bounded, that is,

$$
|S(t)| \leq M, \quad 0<t<T .
$$

Since $A^{-1}$ is compact we note that there exists an arc $C_{n}$ which joints $\mu_{n}$ and some $z_{0}$ with $\operatorname{Re} z_{0}<\inf \left\{\operatorname{Re} \mu_{n}: \mu_{n} \in \sigma(A)\right\}$ and $C_{n}-\left\{\mu_{n}\right\} \subset \rho(A)$ where $\rho(A)$ is the resolvent set of $A$. 
Lemma 2.4. Let $S(t)$ be the semigroup generated by $A$. Then we give an expression of the semigroup that

$$
S(t) g=e^{\mu_{n} t} \sum_{i=0}^{k_{n}-1} \frac{t^{i}}{i !} Q_{n}^{i} g, \quad \forall g \in P_{n} H .
$$

Proof. From the well known fact that

$$
A P_{n}=A \frac{1}{2 \pi i} \int_{\Gamma_{n}}(\mu-A)^{-1} d \mu=\frac{1}{2 \pi i} \int_{\Gamma_{n}} \mu(\mu-A)^{-1} d \mu
$$

we have

$$
S(t) P_{n}=\frac{1}{2 \pi i} \int_{\Gamma_{n}} e^{\mu t}(\mu-A)^{-1} d \mu .
$$

If $g \in P_{n} H$ then $g=P_{n} g$. Hence, we have

$$
\begin{aligned}
S(t) g & =S(t) P_{n} g=\frac{1}{2 \pi i} \int_{\Gamma_{n}} e^{\mu t}(\mu-A)^{-1} g d \mu \\
& =e^{\mu_{n} t} \frac{1}{2 \pi i} \int_{\Gamma_{n}} e^{\left(\mu-\mu_{n}\right) t}(\mu-A)^{-1} g d \mu \\
& =e^{\mu_{n} t}\left\{\sum_{i=0}^{\infty} \frac{t^{i}}{i !}\left(\frac{1}{2 \pi i} \int_{\Gamma_{n}}\left(\mu-\mu_{n}\right)^{i}(\mu-A)^{-1} g d \mu\right)\right\}=e^{\mu_{n} t} \sum_{i=0}^{k_{n}-1} \frac{t^{i}}{i !} Q_{n}^{i} g .
\end{aligned}
$$

Here, we used the nilpotent property of the operator $Q_{n}$ in the last equality. The proof of Lemma is complete.

Remark 3. If the assumption (A) is satisfied then $S(t) P$ is extended to the whole real line so that

$$
S(t) P=\sum_{n=1}^{\infty} \sum_{i=0}^{k_{n}-1} \frac{e^{\mu_{n} t} t^{i}}{i !} Q_{n}^{i}, \quad-\infty<t<\infty .
$$

Let $g \in L^{2}(0, t ; H)$. Then by the assumption (B) for any $\epsilon>0$ there exists a control $v \in L^{2}(0, t ; U)$ such that

$$
\|S(t-\cdot)(g(\cdot)-B v(\cdot))\|_{L^{2}(0, t ; H)} \leq \frac{\epsilon}{2 \sqrt{T}}, \quad 0 \leq t \leq T,
$$

and

$$
\left|v(s)-\sum_{n=1}^{\infty} P_{n} v(s)\right| \leq \frac{\epsilon}{M \sqrt{T}},
$$

in the sense of (3.4). Let us define $h \in H$ by

$$
h=\sum_{n=1}^{\infty} \int_{0}^{t} S(t-s) P_{n} v(s) d s=\sum_{n=1}^{\infty} h_{n} .
$$


Here, we put $h_{n}=\int_{0}^{t} S(t-s) P_{n} v(s) d s$. Since $P_{n} v(s) \in P_{n} H$, in terms of Lemma 3.1 we have that

$$
h_{n}=\int_{0}^{t} S(t-s) P_{n} v(s) d s=\sum_{i=1}^{k_{n}-1} \int_{0}^{t} e^{\mu_{n}(t-s)} \frac{(t-s)^{i}}{i !} Q_{n}^{i} P_{n} v(s) d s .
$$

Define

Then it follows

$$
u(s)=\sum_{n=1}^{\infty} u_{n}(s), \quad u_{n}(s)=P_{n} v(s)
$$

$$
\int_{0}^{t} S(t-s) B u(s) d s=\sum_{n=1}^{\infty} \int_{0}^{t} S(t-s) B u_{n}(s) d s=\sum_{n=1}^{\infty} \int_{0}^{t} S(t-s) P_{n} v(s) d s .
$$

Thus from (3.3) and (3.4) it follows that

$$
\begin{aligned}
& \left|\int_{0}^{t} S(t-s) B u(s) d s-\int_{0}^{t} S(t-s) g(s) d s\right| \\
& \leq\left|\int_{0}^{t} S(t-s) B u(s) d s-\sum_{n=1}^{\infty} \int_{0}^{t} S(t-s) P_{n} v(s) d s\right| \\
& +\left|\sum_{n=1}^{\infty} \int_{0}^{t} S(t-s) P_{n} v(s) d s-\int_{0}^{t} S(t-s) B v(s) d s\right| \\
& +\left|\int_{0}^{t} S(t-s) B v(s) d s-\int_{0}^{t} S(t-s) g(s) d s\right|<\frac{\epsilon}{2}+\frac{\epsilon}{2}<\epsilon .
\end{aligned}
$$

Hence, from (3.1), (3.2) and Hölder inequality it holds

$$
\begin{aligned}
|B u(s)| & \leq\left|\sum_{n=1}^{\infty} B P_{n} v(s)\right| \leq\left|\sum_{n=1}^{\infty} e^{\mu_{n} t-s} \sum_{i=1}^{k_{n}-1} \frac{(t-s)^{i}}{i !} Q_{n}^{i} B P_{n} v(s)\right| \\
& \leq\left|S(t-s) \sum_{n=1}^{\infty} B P_{n} v(s)\right| \leq\left|S(t-s)\left(\sum_{n=1}^{\infty} B P_{n} v(s)-B v(s)\right)\right| \\
& +|S(t-s)(B v(s)-g(s))|+|S(t-s) g(s)| .
\end{aligned}
$$

So from the above equality we can conclude that there exists a positive constant $q$ such that for any $\epsilon>0$

$$
\|B u\|_{L^{2}(0, t ; H)}^{2} \leq q\|g\|_{L^{2}(0, t ; H)}+\epsilon \text {. }
$$

Here, we note the constant $q$ is independent of $g$. Since $\epsilon$ is arbitrary we have proof that the assumption of Theorem 2.3 implies the second statement of (3.1) and so, the condition (3.1) is immediately form the assumption (B). Therefore, in virtue of Theorem 4.1 of [11] the proof of Theorem 2.3 is complete.

Example 1. Assume $U=H$ and $B=1$, which is the identity operator $H$. Then assumption (B) obviously is satisfied. 
Example 2. We consider the heat control system studied by Zhou [10, Example 1] and Naito [5 Example 1]. Let $H=L^{2}(0, \pi)$ and $A=-d^{2} / d x^{2}$ $H=L^{2}(0, \pi)$ and $A=-d^{2} / d x^{2}$ with

$$
D(A)=\left\{y \in H: d^{2} y / d x^{2} \in H \text { and } y(0)=y(\pi)=0\right\} .
$$

Then $\left\{e_{n}=(2 / \pi)^{1 / 2} \sin n x: 0 \leq x \leq \pi, n=1, \ldots\right\}$ is orthonomal base for $H$.

Case 1 Define an infinite dimensional space $U$ by

$$
U=\left\{\sum_{n=2}^{\infty} u_{n} e_{n}: \sum_{n=2}^{\infty} u_{n}^{2}<\infty\right\}
$$

with norm defined by $\|u\|_{U}=\left(\sum_{n=2}^{\infty} u_{n}^{2}\right)^{1 / 2}$. Define a continuous linear operator $B$ from $U$ to $H$ as follows:

$$
B u=2 u_{2} e_{1}+\sum_{n=2}^{\infty} u_{n} e_{n} \text { for } u=\sum_{n=2}^{\infty} u_{n} e_{n} \in U
$$

It is directly seen that the above controller $B$ satisfies the conditions (B). We can also check briefly by using the assumption (3.1). In fact, let $f \in L^{2}(0, T ; H)$ and $f=\sum_{n=1}^{\infty} f_{n}(s) e_{n}$. Then we choose a function $u \in L^{2}(0, t ; U)$ for $0 \leq t \leq T$ such that $u_{2}=\frac{1}{2} f_{1}+f_{2}$ and $u_{n}=f_{n}$ for $n=2,3$, ... Hence, choosing a constant in condition (3.1) such that $q>\frac{7}{2}$, not only the system (1.1) with the operator $A$ mentioned above but also general semilinear case is approximate controllable.

Case 2 Define $U=\left\{e_{1}, \cdots\right\}$. For any $u=\sum_{n=2}^{\infty}$ define

$$
B u=u_{i} e_{1}+\sum_{n=2}^{\infty} u_{n} e_{n},
$$

where $i$ is some fixed integer. Define $P_{U}$ as the orthogonal projection of $H$ on $U$. In this case, $P_{u} B$ is the identity operator in $U$. Hence the assumption (B) is satisfied.

\section{References}

[1] G. Di Blasio, K. Kunisch and E. Sinestrari, $L^{2}$-regularity for parabolic partial integrodifferential equations with delay in the highest-order derivatives, J. Math. Anal. Appl. 102 (1984), 38-57.

[2] J. M. Jeong, Retarded functional differential equations with $L^{1}$-valued controller, Funkcial. Ekvac. 36 (1993), 71-93.

[3] K. Naito, Controllability of semilinear control systems dominated by the linear part, SIAM J. Control Optim. 25 (1987), 715-722.

[4] S. Nakagiri, Structural properties of functional differential equations in banach space, Osaka J. Math. 25 (1988), 353-398.

[5] J. M. Jeong, Y. C. Kwun and J. Y. Park, Approximate controllability for semilinear retarded functional differential equations, J. Dynamics and Control Systems, 5 (1999), 329-346.

[6] H. Tanabe, Functional analysis II, Jikko Suppan Publ. Co., Tokyo, 1981[in Japanese]. 
[7] H. Tanabe, Fundamental solution of differential equation with time delay in Banach space, Funkcial. Ekvac. 35 (1992), 149-177.

[8] K. Yosida, Functional Analysis, 3rd ed., Springer-Verlag Berlin Heidelberg New York, 1980.

[9] H. X. Zhou, Approximate controllability for a class of semilinear abstract equations, SIAM J. Control Optim. 21 (1983), 551-565.

[10] H. X. Zhou, Controllability properties of linear and semilinear abstract control systems, SIAM J. Control Optim. 22 (1984), 405-422.

[11] J. M. Jeong and H. H. Rho, Approximate controllability for semilinear retarded systems, J. Math. Anal. Appl. 321 (2006), 961-975.

Department of Applied Mathematics, Pukyong National University, Busan 608-737, Korea

E-mail address: jmjeong@pknu.ac.kr 